\title{
Ruptura aguda de quiste hidatídico hepático hacia tórax
}

\author{
Acute rupture of a liver hydatid cyst into the thorax \\ Luis Castillo De la Cadena ${ }^{1,2, a}$, Hermes Injante ${ }^{3, a}$, Walter Torres ${ }^{4, a}$, Wilfredo Huamaní ${ }^{\text {,aa }}$, \\ Modesto Carnero ${ }^{3, a}$.
}

\section{RESUMEN}

El quiste hidatídico es una enfermedad parasitaria humana causada por el estado quístico del parasito Echinococcus granulosus. En el Perú hay zonas hiperendémicas de hidatidosis humana. Los quistes hidatídicos hepáticos son los más frecuentes, y la ruptura hacia el tórax es rara, siendo menor de 1\% de los casos. Presentamos el caso de una paciente con quiste hidatídico hepático roto agudamente hacia tórax que es una patología de difícil abordaje y de alto riesgo para el paciente y como fue resuelto en forma multidisciplinaria.

PALABRAS CLAVE: Quiste hidatídico, diagnóstico, cirugía, hígado, equinococosis. (Fuente: DeCS BIREME).

\section{SUMMARY}

Hydatid cyst is a human parasitic disease caused by the cystic stage of Echinococcus granulosus. There are hyperendemic areas of hydatidosis in Peru. Liver involvement is the common affection of the disease, rupture of liver cysts into the thorax is rare (less than $1 \%$ of cases). We present the case of a female patients who presented with a liver cyst rupture to the thorax, a multidisciplinary approach was needed to cure the patient.

KEYWORDS: Hydatid cyst, diagnostic, surgery, liver, echinoccocosis. (Source: MeSH NLM).

\section{INTRODUCCIÓN}

El quiste hidatídico es una enfermedad parasitaria humana causada por el estado quístico del parasito Echinococcus granulosus, el cual infecta el intestino de los perros, su huésped definitivo. El humano sirve como huésped incidental por ingestión de huevos en vegetales o agua contaminada con las heces del perro. La enfermedad hidatídica es endémica en Sudamérica especialmente donde existe crianza de ganado ovino (1-3).
En el Perú la prevalencia de hidatidosis humana es 7 - 11 / 100000 habitantes. Sin embargo, hay regiones de alta prevalencia, como en Junín, Huancavelica, Pasco y Ayacucho (2,3).

La hidatidosis es considerada hiperendémica en el departamento de Pasco. La incidencia anual para el año 2000 fue 78,6 x 100 000. La tasa letalidad de pacientes hospitalizados por el daño se estima entre 1 y $12 \%(2,3)$ pero un estudio encontró una tasa de $8.8 \%$ en dicha región (1).

\footnotetext{
Servicio de Cirugía de Tórax y Cardiovascular, Hospital María Auxiliadora. Lima, Perú.

Unidad de Epidemiología Clínica, Facultad de Medicina Alberto Hurtado, Universidad Peruana Cayetano Heredia. Lima, Perú.

Servicio de Cirugía General, Hospital María Auxiliadora. Lima, Perú.

Servicio de Neumología, Hospital María Auxiliadora. Lima, Perú.

Servicio de Cardiología del Hospital María Auxiliadora. Lima, Perú.

Médico Asistente
} 
Los quistes hidatídicos comúnmente se localizan en el hígado ( $>65 \%$ de los casos) y en los pulmones $(25 \%)$. El quiste hidatídico hepático puede sufrir ruptura hacia nivel peritoneal (lo más frecuente) y raramente se produce ruptura hacia la cavidad torácica, y constituyen el $0.6 \%$ de los casos (4). $\mathrm{La}$ asociación con una fístula bilio-bronquial es un escenario complejo por su difícil manejo y alto riesgo de morbi-mortalidad para el paciente (4-7).

El objetivo de este reporte, fue describir el caso de una paciente con quiste hidatídico hepático con ruptura abrupta hacia el tórax; revisar su presentación clínica, la presencia de fístula bilio bronquial y el compromiso pulmonar y pericárdico, los métodos diagnósticos utilizados y el manejo médico-y quirúrgico multidisciplinario de esta patología de alta morbi-mortalidad.

\section{PRESENTACIÓN DEL CASO}

Mujer de 41 años procedente de Chaupimarca, Pasco; quién se encontraba de tránsito por Lima; acudió a emergencia del Hospital María Auxiliadora por dolor torácico derecho intenso y taquipnea de 10 horas de evolución, de inicio brusco y curso progresivo. $\mathrm{Al}$ ingreso: Presión Arterial: 100/60, saturación de $\mathrm{O}_{2}$ : $96 \%$, frecuencia respiratoria 30 por minuto, pulso 129 por minuto, peso $45 \mathrm{~kg}$ y una talla de $1,45 \mathrm{~m}$. Al examen de tórax, se encontró abolición del murmullo vesicular en $2 / 3$ del hemitórax derecho y matidez en la base de campo pulmonar derecho.

El hemograma mostró leucocitosis de 15300 con $0 \%$ de abastonados y el análisis de gases arteriales $\mathrm{PaO}_{2} / \mathrm{FiO}_{2} 355$.

La radiografía de tórax al ingreso mostró velamiento basal derecho. La ecografía abdominal reportó una masa de $10,3 \times 12,3 \times 12,7 \mathrm{~cm}$ en los segmentos V, VI y VII del hígado, volumen de $853,4 \mathrm{ml}$ compatible con quiste hidatídico tipo IV CE4 en la clasificación de Garbhi y OMS, respectivamente.

La tomografía espiral multicorte (TEM) abdomino torácica mostró una tumoración quística bilobulada en la base de hemitórax y región subfrénica del lado derecho; además, un quiste hidatídico hepático con comunicación a la cavidad pleural (figura 1), un área de condensación con zona de abscedación comprometiendo el lóbulo medio, atelectasia pasiva del lóbulo inferior derecho y moderado derrame pleural (figura 2).

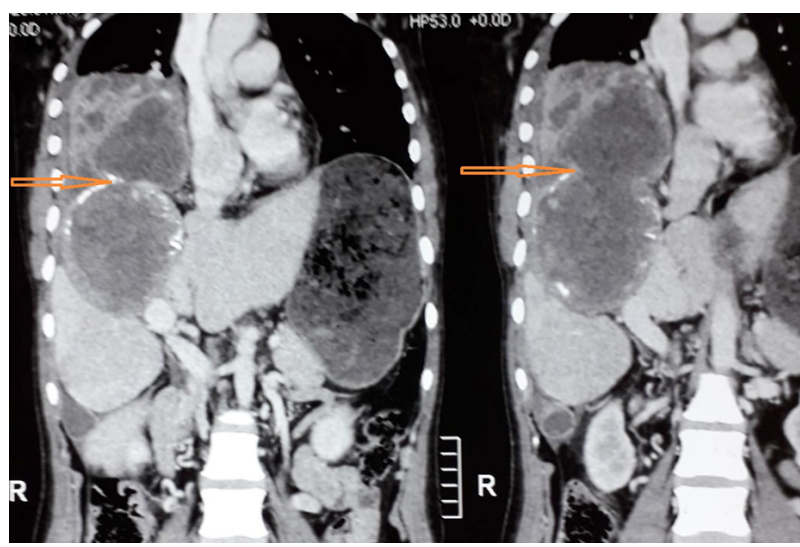

Figura 1. Tomografía espiral multicorte abdomino torácica muestra la cavidad quística hepática con signos de calcificación y que conecta a la cavidad torácica produciendo atelectasia y áreas de abscedación del lóbulo inferior derecho.

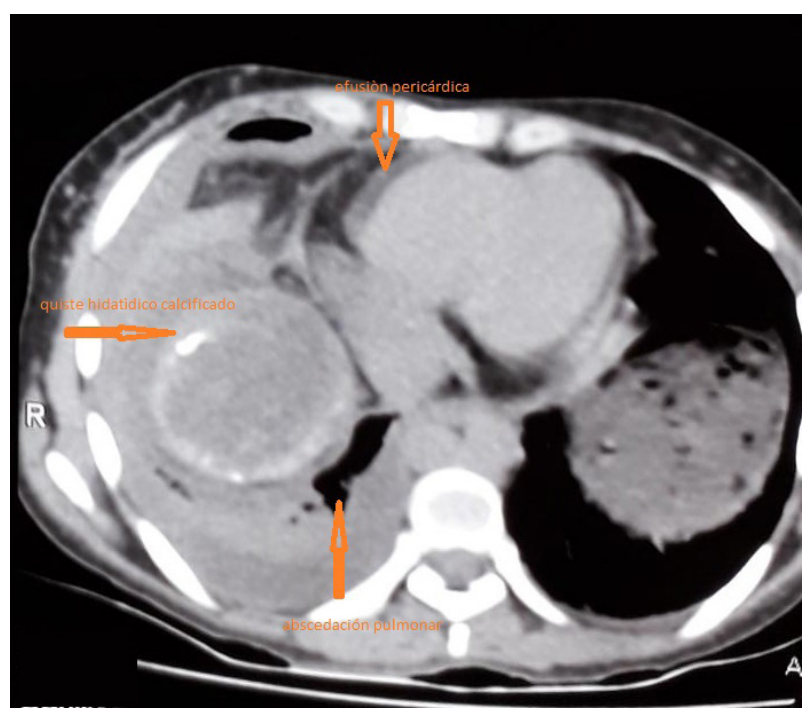

Figura 2. TEM torácica muestra la cavidad quística hepática con calcificaciones, que produce atelectasia y áreas de abscedación del lóbulo inferior derecho. Además, se evidencia derrame pleural y pericárdico.

El ecocardiograma mostró cavidades normales; motilidad regional y global del VI conservada; FEVI 63\%; función sistólica del VD conservada TAPSE $<18 \mathrm{~mm}$, derrame pericárdico a predominio de pared posterior de $210 \mathrm{ml}$ aproximadamente, sin signos de taponamiento y abundante fibrina.

El diagnóstico de ingreso fue ruptura aguda de quiste hidatídico hepático hacia cavidad pleural; en el tránsito a sala de operaciones presentó un episodio de biliptisis escasa, lo que produjo una saturación de oxígeno intraoperatoria menor de $90 \%$. 
Entre los hallazgos intraoperatorios más significativos se encontró líquido inflamatorio de aprox. $1000 \mathrm{ml}$, y adherencias múltiples en pleura parietal, mediastinal y diafragma; perforación diafragmática de aprox. $10 \mathrm{~cm}$ que comunicaba con la cavidad quística complicada, perforación pulmonar en la base de lóbulo inferior derecho que comunicaba a la cara lateral (segmento 6) y perforación bronquial en hilio lobar inferior derecho. Además, quiste hidatídico hepático en varios estadios de maduración fragmentados y pericardio de características normales con derrame no significativo.

El abordaje fue por toracotomía antero lateral procediéndose a la evacuación de líquido inflamatorio a nivel pleural; luego, se procedió a abordar la cavidad creada entre el quiste hepático y el lóbulo inferior derecho, una colección serofibrinosa. Se ubicó la fístula bronquial a nivel del hilio y se procedió a su cierre, luego se procedió a la rafia del lóbulo inferior derecho que se encontraba perforado y comunicaba los segmentos inferiores al segmento apical (segmento 6), dado que el diafragma estaba perforado se procedió a evacuación del quiste hidatídico hepático complicado y posteriormente se procedió a cerrar el diafragma con sutura no reabsorbible (figura 3). Se colocaron dos drenes torácicos y un dren de Jackson Pratt a nivel de cavidad quística hepática.

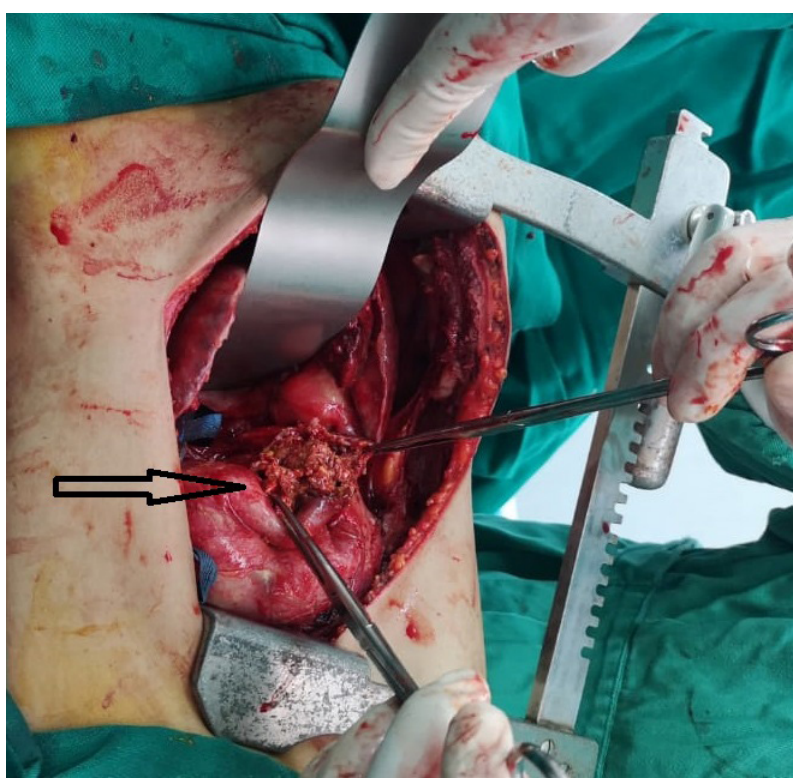

Figura 3. Toracotomía antero lateral muestra la perforación diafragmática y los restos del quiste hidatídico hepático (flecha). La valva superior oculta parcialmente la zona de pulmón perforado y de la fístula bronquial.
En el post operatorio inmediato, la paciente evolucionó con insuficiencia respiratoria requiriendo ventilación mecánica, debido a neumonía contralateral por aspiración (biliptisis).

Desde el segundo día post operatorio la paciente presentó una mejoría clínica espectacular, con rehabilitación pulmonar y ventilación no invasiva. El derrame pericárdico fue evaluado diariamente con ecocardiograma con valores en disminución de $210 \mathrm{ml}$ al inicio a menos de $100 \mathrm{ml}$ al quinto día post operatorio. Los drenes torácicos fueron retirados al decimoquinto día, permaneciendo con dren de Jackson Pratt, por presentar fistula biliar de bajo débito (menor de $300 \mathrm{ml} /$ día), la cual resolvió mediante la instilación de yodopovidona.

\section{DISCUSIÓN}

El contacto íntimo del quiste hidatídico hepático con el diafragma favorece el cruce de la barrera diafragmática con ruptura de estos quistes en la pleura o en un lóbulo pulmonar. Así, el tránsito intratorácico de estas lesiones depende de varios factores: el contacto íntimo del quiste en la cúpula hepática con la cúpula diafragmática; la gradiente toraco-abdominal que tiende a aspirar el contenido del quiste hidatídico del hígado hacia la cavidad torácica; la erosión del diafragma por isquemia-necrosis causada por la posible superinfección del quiste y los fenómenos inflamatorios, y la corrosión de todos los tejidos en contacto con la bilis (8).

El quiste, después de haber pasado por el diafragma, puede provocar una fístula en los bronquios, generalmente de los lóbulos bajos o lóbulo medio, raramente en el lóbulo superior o el de la língula. La bilis causa por acción cáustica en el pulmón o el árbol bronquial, lesiones que van desde la neumonía hidatídica simple hasta la constitución de una caverna, o incluso a la destrucción total del parénquima pulmonar (9).

En la fisiopatología del compromiso respiratorio juega un rol importante la neumonitis química producida por la bilis a lo cual puede sumarse la infección bacteriana $(6,7,10)$.

En el caso de esta paciente, la bilis perforó el hilio lobar inferior derecho produciendo fistula bilio bronquial y perforación pulmonar del lóbulo inferior derecho dejando una caverna extensa y neumonitis aspirativa por biliptisis. 
Tabla 1. Migración torácica del quiste hidatídico hepático.

TIPO I: fistulización directa del quiste en los bronquios

-IA: fístula bronquial de pequeño tamaño

-IB: fístula bronquial de gran calibre

\section{TIPO II: caverna intrapulmonar}

-IIA: sin fístula bronquial o fístula bronquiolar

-IIB: con fístula bronquial grande

\section{TIPO III: Colección intermedia intrapleural enquistado}

-IIIA: sin fístula bronquial

-IIIB: con fístula bronquial

-IIIC: con fístula en la pared

\section{TIPO IV: ruptura en la cavidad pleural grande}

IVA: ruptura aguda: pleuritis biliohidática

-IVB: hidatidosis pleural secundaria

Debido a la variación de lesiones que se producen en el tránsito al tórax existe la clasificación de Mestiri et al. (5). Esta clasificación tiene 4 tipos, los que se muestran en la tabla 1.

El caso presentado se clasifica como tipo III B porque había colección entre el quiste y el lóbulo inferior derecho (que también estaba perforado) pero llegó hasta fistula bronquial.

Las consecuencias de la rotura son de dos órdenes: primarias, que engloban los fenómenos inmediatos, tanto de carácter alérgico o anafiláctico, debido a la reabsorción de líquido hidatídico por la serosa pleural cuando aquél se vierte en la misma (y cuya intensidad es muy variable dependiendo del estado de sensibilidad del paciente), como de carácter mecánico-infeccioso inherentes a la propia perforación (neumotórax, hidroneumotórax y aún pleuresía purulenta si el quiste esta previamente infectado; procesos que afectan al conjunto de la gran cavidad pleural cuando ésta está libre, y son más o menos localizados cuando existe sínfisis en alguna zonal, y secundarias, condicionadas de una parte por la evolución del parásito, y de otra, por la posible y fácil infección subsiguiente a la rotura, comprendiendo así tanto la equinococosis pleural primitiva heterotópica y secundaria como el pioneumotórax, complicación más o menos tardía $(9,11)$.

La elección del abordaje es siempre una cuestión de controversia. De hecho el máximo confort y seguridad es obtenido con la toracotomía debido a las lesiones importantes del parénquima pulmonar, a menudo requiriendo una resección pulmonar como lobectomía o segmentectomía. La frenicoplastia es posible después de la resección de todas las partes fibrosadas y liberación del saco costo-diafragmático (7).

La fístula biliar externa es la complicación más frecuente de la cirugía del quiste hidatídico hepático complicado a tórax variando según los distintos estudios entre el 6 y 28\%; aunque la gran mayoría cierra espontáneamente, esta puede persistir en algunos casos durante largo tiempo $(8,9)$, como fue nuestro caso que duró por 45 días.

En el caso del compromiso pericárdico, se limitó a una irritación por contigüidad provocando derrame que después de la cirugía fue disminuyendo progresivamente. Pero el efecto corrosivo de la bilis puede dar mayor compromiso pericárdico con perforación y embolismo en la vena cava inferior hacia arterias pulmonares $(8,12)$.

La ruptura de un quiste hidatídico hacia la cavidad pericárdica puede dar lugar a un derrame pericárdico, que es un proceso inflamatorio alérgico (8). El derrame puede ser masivo y causar taponamiento cardíaco. En nuestro caso, al no existir evidencia de taponamiento, no fue intervenido. Además, una intervención de un derrame pericárdico debido a una enfermedad hidatídica puede agravar aún más el derrame ya que se sabe que una pericardiotomía reactiva la inflamación pericárdica (8).

El manejo multidisciplinario redunda en mejores resultados en favor del paciente y se traduce en una menor morbilidad y estancia hospitalaria. 
En el plano terapéutico, la intervención quirúrgica precoz constituye el único tratamiento radical efectivo (9).

\section{Declaración de conflictos de interés:}

Los autores declaran no tener conflictos de interés.

\section{Contribución de autoría:}

Los autores participaron en el seguimiento del paciente y contribuyeron con información para la elaboración del artículo. Todos los autores leyeron y aprobaron el informe final.

\section{Correspondencia}

Luis Castillo De la Cadena

Correo electrónico: medicoluis@hotmail.com

\section{REFERENCIAS BIBLIOGRÁFICAS}

1. Nunez, E, Calero D, Estares L, Morales A. Prevalencia y factores de riesgo de hidatidosis en población general del distrito de Ninacaca-Pasco, Perú 2001. An Fac Med. 2003; 64(1): 34-42.

2. Náquira $\mathrm{C}$, Bullón $\mathrm{F}$, Balvín $\mathrm{G}$, Reyes $\mathrm{N}$. Epidemiología de la hidatidosis en el Perú. En: Ministerio de Salud. Anales del Seminario Nacional de hidatidosis y enfermedades de transmisión alimentaria. Lima: MINSA;1989. p 122-137.

3. Náquira C. Situación de la hidatidosis en el Perú. Washington DC: Reunión del grupo científico de trabajo sobre hidatidosis; Organización Panamericana de la Salud;1994. p. 262-77.

4. Msaad S, Yangui I, Ketata W, et al. Hydatid cysts of the liver ruptured into the thorax (about five cases). Rev pneumol clin. 2015; 75(5):255-63.
5. Gomez R, Moreno E, Loinaz C, et al. Diaphragmatic or transdiaphragmatic thoracic involvement in hepatic hydatid disease: surgical trends and classification. World J Surg. 1995;19(5):714-9.

6. Rabiou S, Lakranbi M, Ouadnouni Y, Panaro F, Smahi M. Surgical management of hydatid Biliobronchial fistula by exclusive thoracotomy. Int J Surg. 2017; 41:112-18.

7. Rabiou S, Harmouchi H, Belliraj L, et al. Management for ruptured liver hydatid cysts in the chest: Experience of a Moroccan Center. Clin Surg. 2017; 2:1757.

8. Ahuja S, Karande S, Koteyar S, Kulkarni M. Hepatic hydatid cyst rupturing into sub-diaphragmatic space and pericardial cavity. J Postgrad Med. 2001; 47:37.

9. Ramos G, Duque J, Yuste J, et al. La ruptura intrapleural del quiste hidatidico de pulmon. Arch Bronconeumol. 1984; 20(2):47-54. doi:10.1016/ s0300-2896(15)32256-0

10. Losada H, Vial M, Manterola C, Pineda V. Fístula biliobronquial secundaria a quiste hepático hidatídico en tránsito al tórax: Reporte de caso. Rev Chil Cir. 2006; 58(3):224-227.

11. Munoz C, Burgos L, Fonseca V. Fístula biliobronquial de origen hidatídico. Rev Chil Cir. 2009; 61(6):504506.

12. Poyraz N, Demirbaş S, Korkmaz C, Uzun K. Pulmonary embolism originating from a hepatic hydatid cyst ruptured into the inferior vena cava: $\mathrm{CT}$ and MRI findings. Case Rep Radiol. 2016; 2016:3589812. doi: $10.1155 / 2016 / 3589812$

Recibido: 25/02/2019

Aceptado: 28/03/2019 